\title{
EDITORIAL
}

\section{Multimodality measurements of small airways disease}

\author{
G.G. King*,\#,ף and C.M. Salome ${ }^{\#, \tau_{9}+}$
}

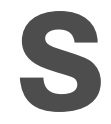

mall airways disease has long been recognised as being physiologically important in obstructive lung diseases, particularly in chronic obstructive pulmonary disease (COPD) [1]. The study of Cosio et al. [2], in which the severity of small airway inflammation and remodelling in smokers correlated with airway closure and ventilation maldistribution, as measured by single breath washout tests, is a landmark one because it linked small airways tests with pathology. Furthermore, these parameters of small airway function were more sensitive than spirometry for the presence of small airways disease. Results of more recent studies, in which small airway function was measured by the newer multiple breath nitrogen washout, showed that small airway function becomes abnormal after only 10 pack-yrs smoking, whereas spirometry becomes abnormal after 20 pack-yrs [3]. In asthma, however, the function of the small airways has been studied much less, perhaps due to the paucity of resected lung specimens on which correlations can be made between measurements of small airway function and pathology. The disease-related changes to the structural components of the airways in asthma involve both small and large airways [4]. These pathological changes, often referred to as remodelling, lead to thickening of the walls of the small airways. This is a feature that seems particularly characteristic of asthma as opposed to COPD [5].

There is now an increasing body of evidence suggesting that small airways disease in asthma has important clinical consequences, to which the study by ZEIDLER et al. [6], in this issue of the European Respiratory Journal, makes an important contribution. In the study by ZEIDLER et al. [6], high-resolution computed tomography (HRCT) was acquired in asthmatic patients before and after methacholine challenge tests, which were then repeated after 4-weeks' treatment with either montelukast or placebo, in a randomised, double-blind fashion. Their finding that montelukast reduced gas trapping in expiratory HRCT scans compared with placebo was accompanied by two other important observations; the reduction in gas trapping associated with montelukast treatment was tightly correlated with the improvement in overall quality of life, but it did not correlate with any physiological measures, in particular, airway hyperresponsiveness (AHR) and closing volumes measured by single-breath nitrogen washout. The mechanisms that underlie this relationship are not clear,

\footnotetext{
*The Dept of Respiratory Medicine, Royal North Shore Hospital, St Leonards, "The Woolcock Institute of Medical Research, and ${ }^{+}$Dept of Medicine, University of Sydney, Sydney, and "The Cooperative Research Centre for Asthma and Airways, Camperdown, Victoria, Australia.

CORRESPONDENCE: G.G. King, Woolcock Institute of Medical Research, Dept of Respiratory Medicine, Royal North Shore Hospital, St Leonards, New South Wales, Australia. Fax: 61 299066391. E-mail: ggk@woolcock.org.au
}

but to the extent that visualised gas trapping is highly likely to be due to small airway disease, it suggests that small airway disease may influence the clinical manifestations of asthma. An increased risk of severe exacerbations associated with increased closing volumes, reported in a more severe group of asthmatics, supports this notion [7].

The absence of any relationship between gas trapping and other physiological measures of asthma in the study by ZEIDLER et al. [6], particularly AHR, raises other questions, but may have been because the patients had clinically mild-tomoderate asthma and, in terms of spirometry, lung volumes and closing volumes, had normal physiology. Additionally, the information about the small airways garnered from expiratory HRCT and lung function parameters are complementary and may not necessarily correlate. The closing volume is a volume at which a rapid increase in airway closure occurs, whereas imaging indicates the extent of lung that is affected by the closure. Two individuals with a similar proportion of lung affected by airway closure and gas trapping could have greatly differing closing volumes, depending on when those airways closed during the expiration towards residual volume [8]. Another problem with the closing volume measurement from single breath washouts is that a closing point cannot be discerned from the washout, particularly when ventilation distribution is highly abnormal leading to a steep alveolar slope. In the study by ZEIDLER et al. [6], closing volume could not be measured in $35 \%$ of washouts. Their use of computerised analyses of their HRCT data, however, is noteworthy and, along with similar approaches in other studies, demonstrates the potentially useful measurements that can be derived from HRCT image data. Although at present used only for research applications, such objective quantification is likely to become routine in clinical radiological practice as an aid to diagnosis and monitoring.

Probing the peripheral airways in asthma has always been difficult. There are many potential published methods which include: forced oscillation technique (FOT) and multiple breath nitrogen washout test [9]; single breath nitrogen washout [7]; frequency dependence of compliance [10]; maximal flow during helium-oxygen breathing [11]; peripheral wedged catheters [12]; positron emission tomography (PET) [13]; and magnetic resonance imaging [14]. The underlying basis of the noninvasive methods is that small airways disease leads to either abnormal ventilation distribution and/or greater airway closure [15] and it would be reasonable to expect that they are indeed interrelated. It should be possible to use HRCT to derive an index of ventilation heterogeneity and xenonenhanced computed tomography has been used in such a way to measure ventilation distribution [16]. Quantification of 
the variability of lung attenuation, i.e. a mosaic perfusion pattern of the lung parenchyma, could be a meaningful measure of small airway disease and this needs further investigation against other parameters of ventilation distribution. Airway narrowing, when measured from HRCT, has been shown to be heterogeneous in asthmatics in response to methacholine [17], but the resolution is such that direct visualisation of the small airways is very limited and most of the heterogeneity can be seen in larger airways. Unless resolution greatly increases without a concomitant increase in radiation exposure, direct visualisation of the small airways with HRCT will remain limited. Alternatively, although HRCT measurements of airway dimensions as markers of remodelling are predominantly of medium-sized airways, they are in fact representative of the degree of remodelling in small airways determined by pathology [18].

Imaging clearly has an important role in the study of small airways, but because of the radiation exposure associated with nuclear imaging and HRCT, it is unlikely to be used in a routine clinical setting. However, when combined with lung function measurements and with computational modelling, imaging can be a powerful approach to investigating small airway function in asthma. An approach was used in which forced oscillation measurements of lung resistance and elastance, and ventilation distribution derived from PET imaging, were interpreted, with the aid of a complex lung model, to study airway narrowing in asthma [13, 19]. The results of the model interpretation were that, although the ventilation defects and inhomogeneities were fairly macroscopic, suggesting closure and severe narrowing in large airways, the basis of these "large scale" defects was likely to be heterogeneity and closure in small airways.

FOT is a practical method of measurement that could potentially be used in routine clinical practice to measure small airway function because of the ease of administration of the test and, potentially, production of relatively cheap devices. Such devices would be capable of producing $4-\mathrm{Hz}$ oscillations, which should reflect the function in the small conducting airways. This is supported by the fact that respiratory conductance derived from FOT correlates with ventilation heterogeneity in small airways measured by multiple breath nitrogen washout, as well as with airway closure [9]. Respiratory elastance derived from FOT may also potentially provide very useful information, both clinically and in terms of mechanisms, in that it is particularly sensitive to airway closure. This represents another area of small airway function in asthma that deserves closer attention.

Given the likely clinical importance of small airways disease in asthma, such as in exacerbations and in fixed flow obstruction, where patients behave like chronic obstructive pulmonary disease, more studies, like the landmark study by Cosio et al. [2], that relate small airway parameters to other important pathological and physiological outcomes, particularly airway hyperresponsiveness, are needed. Another aspect raised in the study by ZEIDLER et al. [6] is the principle that oral antiinflammatory medication, in this case montelukast, might improve small airway function. This raises the question of whether treating small airways disease requires specific therapy other than conventional inhaled therapy which, because of the typical size distribution of the inhaled particles of $\sim 5 \mu \mathrm{m}$, means that they are unlikely to deposit in peripheral airways. The greater availability of and rapid technological improvements in tools for measuring small airways means that they are becoming less and less the "silent zone of the lung".

\section{REFERENCES}

1 Hogg JC, Macklem PT, Thurlbeck WM. Site and nature of airway obstruction in chronic obstructive lung disease. $N$ Engl J Med 1968; 278: 1355-1360.

2 Cosio M, Ghezzo H, Hogg JC, et al. The relations between structural changes in small airways and pulmonaryfunction tests. N Engl J Med 1978; 298: 1277-1281.

3 Verbanck S, Schuermans D, Meysman M, Paiva M, Vincken W. Noninvasive assessment of airway alterations in smokers: the small airways revisited. Am J Respir Crit Care Med 2004; 170: 414-419.

4 Carroll N, Elliot J, Morton A, James A. The structure of large and small airways in nonfatal and fatal asthma. Am Rev Respir Dis 1993; 147: 405-410.

5 Kuwano K, Bosken C, Pare P, Bai T, Wiggs B, Hogg J. Small airways dimensions in asthma and in chronic obstructive pulmonary disease. Am Rev Respir Dis 1993; 148: 1220-1225.

6 Zeidler MR, Kleerup EC, Goldin JG, et al. Montelukast improves regional air-trapping due to small airways obstruction in asthma. Eur Respir J 2006; 27: 307-315.

7 in 't Veen JC, Beekman AJ, Bel EH, Sterk PJ. Recurrent exacerbations in severe asthma are associated with enhanced airway closure during stable episodes. Am J Respir Crit Care Med 2000; 161: 1902-1906.

8 King GG, Eberl S, Salome CM, Young IH, Woolcock AJ. Differences in airway closure between normals and asthmatics measured by SPECT and Technegas. Am J Resp Crit Care Med 1998; 158: 1900-1908.

9 King GG, Downie SR, Verbanck S, et al. Effects of methacholine on small airway function measured by forced oscillation technique and multiple breath nitrogen washout in normal subjects. Respir Physiol Neurobiol 2005; 148: 165-177.

10 Woolcock AJ, Vincent NJ, Macklem PT. Frequency dependence of compliance as a test for obstruction in the small airways. J Clin Invest 1969; 48: 1097-1106.

11 Despas PJ, Leroux M, Macklem PT. Site of airway obstruction as determined by measuring maximal expiratory flow breathing air and a helium-oxygen mixture. J Clin Invest 1972; 51: 3235-3243.

12 Kaminsky DA, Bates JH, Irvin CG. Effects of cool, dry air stimulation on peripheral lung mechanics in asthma. Am J Respir Crit Care Med 2000; 162: 179-186.

13 Venegas JG, Winkler T, Musch G, et al. Self-organized patchiness in asthma as a prelude to catastrophic shifts. Nature 2005; 434: 777-782.

14 Samee S, Altes T, Powers P, et al. Imaging the lungs in asthmatic patients by using hyperpolarized helium-3 magnetic resonance: assessment of response to methacholine and exercise challenge. J Allergy Clin Immunol 2003; 111: 1205-1211.

15 Macklem PT. The physiology of small airways. Am J Respir Crit Care Med 1998; 157: 181S-183S. 
16 Kreck TC, Krueger MA, Altemeier WA, et al. Determination of regional ventilation and perfusion in the lung using xenon and computed tomography. J Appl Physiol 2001; 91: 1741-1749.

17 King GG, Carroll JD, Muller NL, et al. Heterogeneity of narrowing in normal and asthmatic airways measured by HRCT. Eur Respir J 2004; 24: 211-218.
18 Nakano Y, Wong JC, de Jong PA, et al. The prediction of small airway dimensions using computed tomography. Am J Respir Crit Care Med 2005; 171: 142-146.

19 Tgavalekos NT, Tawhai M, Harris RS, et al. Identifying airways responsible for heterogeneous ventilation and mechanical dysfunction in asthma: an image functional modeling approach. J Appl Physiol 2005; 99: 2388-2397. 